\title{
Statistical Tauberian theorems for Cesàro integrability mean based on post-quantum calculus
}

Received: 22 December 2019 / Accepted: 16 April 2020 / Published online: 20 May 2020

(C) The Author(s) 2020

\begin{abstract}
The notion of statistical convergence is more general than the classical convergence. Tauberian theorems via different ordinary summability means have been established by many researchers. In the present work, we have established some new Tauberian theorems based on post-quantum calculus via statistical Cesàro summability mean of real-valued continuous function of one variable under oscillating behavior and De la vallée Poussin mean of a single integral. Moreover, some remarks and corollaries are provided here to support our theorems.
\end{abstract}

Mathematics Subject Classification $\quad 40 \mathrm{C} 10 \cdot 40 \mathrm{G} 05 \cdot 40 \mathrm{E} 05$

\section{Introduction and motivation}

It makes no sense to speak of the sum of a divergent series. Nevertheless, a series that is not "too badly divergent" can be assigned a generalized sum in a variety of natural ways. We are familiar with the notion of summability in connection with the theorems of Abel and Cesàro, which asserts that every convergent series is Abel or Cesàro summable to its ordinary sum. More generally, an Abelian theorem or Cesàro theorem work to the effect that a method of summability assigns to each convergent series its ordinary sum. A Tauberian theorem goes in the opposite direction and asserts that every summable series, which is not too badly divergent is actually convergent.

Tauber [33] introduced the first Tauberian theorem for single sequence, that an Abel summable sequence is convergent with some suitable conditions. A number of authors such as Landau [18], Hardy and Littlewood [7], and Schmidt [25] obtained some classical Tauberian theorems for Cesàro and Abel summability methods of single sequence. Recently, Çanak and Totur [2], and Jena et al. [9] investigated and studied several Tauberian theorems for single sequence. Knopp [16] obtained some classical type Tauberian theorems for Abel and $(C, 1,1)$ summability methods of double sequences and proved that these methods hold for the set of bounded sequences. Móricz [19] proved some Tauberian theorems for Cesàro summable double sequences and deduced Tauberian theorems of Landau [17] and Hardy [6] type. Recently, Totur [34] extended some classical type Tauberian theorems for single and double sequences in connection with one-sided Tauberian theorems.

P. Parida

Department of Mathematics, Kuntala Kumari Sabat Women's College, Balasore, Odisha 756003, India

E-mail: priyadarsiniparida1@gmail.com

S. K. Paikray $(\varangle) \cdot$ B. B. Jena

Department of Mathematics, Veer Surendra Sai University of Technology, Burla, Odisha 768018, India

E-mail: skpaikray_math@vssut.ac.in

B. B. Jena

E-mail: bidumath.05@gmail.com 
Furthermore, Çanak and Totur [1] proved a Tauberian theorem for Cesàro summability of single integral and also, they established the alternative proofs of some classical type Tauberian theorems for the Cesàro summability of single integral. Moreover, in the year 2017, Jena et al. [11] proved the Tauberian theorems for Harmonic summability of double-integrable real-valued function over $\mathbb{R}^{2}$ and also established the inclusion relation between the statistical convergence and classical convergence. Very recently, Çanak et al. [3] introduced and studied the concept of Tauberian theorem for Cesàro integrability mean based upon quantum calculus via usual convergence.

Motivated essentially by the above-mentioned investigations and results, here we prove the statistical versions of Tauberian theorems via Cesàro integrability mean based upon post-quantum calculus of a realvalued continuous function of one variable under slow oscillation and De la Vallée Poussin mean of the integral. In fact, we extend here the result of Çanak [3] using the idea of Tauberian theorems for Cesàro integrability mean based on quantum calculus via usual summability.

\section{Preliminaries and definitions}

The notion of statistical convergence was first introduced and studied by Fast [5] and Steinhaus [32] independently. For more recent works in this direction, one may refer [4,8-15,21-24,26-31].

Let $f(x)$ be a function in $\mathbb{R}$ with the partial sum

$$
s(x)=\int_{0}^{x} f(\zeta) \mathrm{d} \zeta, \quad(0<x<\infty) .
$$

The $(C, 1)$ mean of $f(x)$ is (see [20]),

$$
\sigma(s(x))=\frac{1}{x} \int_{0}^{x} s(\zeta) \mathrm{d} \zeta
$$

The integral $\int_{0}^{x} s(\zeta) \mathrm{d} \zeta$ is $(C, 1)$-summable to a finite number $\ell$, if

$$
\lim _{x \rightarrow \infty} \sigma(s(x)) \rightarrow \ell
$$

The integral $\int_{0}^{x} s(\zeta) \mathrm{d} \zeta$ is statistically $(C, 1)$-summable to a finite number $\ell$ if, for each $\epsilon>0$,

$$
\lim _{u \rightarrow \infty} \frac{1}{u} \mid\{0<x \leqq u \text { and }|\sigma(s(x))-\ell| \geqq \epsilon\} \mid=0 .
$$

In this case, we write

$$
\text { stat } \lim _{x \rightarrow \infty} \sigma(s(x))=\ell
$$

If $\lim _{x \rightarrow \infty} s(x)=\ell$ exists, then the relations (2.1) and (2.2) hold. However, in general, the converse is not true. To prove the sufficient part, we use the oscillatory behavior and De la vallée Poussin mean of the above integral over $\mathbb{R}$. Such a condition is called a Tauberian condition and the resulting theorem is called a Tauberian theorem.

Quantum calculus (or $q$-calculus) is the modern name for the investigation of calculus which is focused on the idea of deriving $q$-analogues of results belonging to standard calculus without using limits. The $q$-calculus served as a bridge between mathematics and physics. Furthermore, there is a possibility of extension of the $q$-calculus to post-quantum calculus (or $(p, q)$-calculus). It has gained noticeable importance and popularity during the past 3 decades due mainly to its applications in different mathematical areas such as number theory, combinatorics, orthogonal polynomials, basic hypergeometric series and other sciences such as statistical mechanics, quantum theory, and the theory of relativity.

We recall some basic concepts of $(p, q)$-calculus and some associated properties of $(p, q)$-Cesàro integrability mean method. For any $n \in \mathbb{N}$, the $(p, q)$-integer $[n]_{p, q}$ is defined by

$$
[n]_{p, q}= \begin{cases}\frac{p^{n}-q^{n}}{p-q} & (n \geq 1) \\ 0 & (n=0)\end{cases}
$$


where $0<q, \quad p \leq 1$.

The $(p, q)$-factorial is defined by

$$
[n] !_{p, q}= \begin{cases}{[1]_{p, q}[2]_{p, q} \ldots[n]_{p, q}} & (n \geq 1) \\ 1 & (n=0) .\end{cases}
$$

The $(p, q)$-binomial coefficient is defined by

$$
\left[\begin{array}{l}
n \\
k
\end{array}\right]_{p, q}=\frac{[n] !_{p, q}}{[k] !_{p, q}[n-k] !_{p, q}} \text { for all } n, k \in \mathbb{N} \text { and } n \geq k .
$$

We also recall that, suppose $0<q<p \leqq 1$ and the $(p, q)$-derivative of the function $f$ is defined as

$$
D_{p, q} f(x)=\frac{f(p x)-f(q x)}{(p-q) x}, x \neq 0 .
$$

As a special case, when $p=1,(p, q)$-derivative reduces to $q$-derivative and also, the $(p, q)$-derivative fulfills the following product derivative properties:

$$
D_{p, q}(f(x) g(x))=f(p x) D_{p, q} g(x)+g(q(x)) D_{p, q} f(x)
$$

and

$$
D_{p, q}(f(x) g(x))=g(p x) D_{p, q} f(x)+f(q(x)) D_{p, q} g(x) .
$$

Next, let $f$ be a real-valued continuous function and let $a$ be a real number. The $(p, q)$-integral of $f(x)$ on $[0, a]$ is defined as

$$
\int_{0}^{a} f(x) \mathrm{d}_{p, q} x=(p-q) a \sum_{k=0}^{\infty} \frac{q^{k}}{p^{k+1}} f\left(\frac{q^{k}}{p^{k+1}} a\right), \quad \text { if } \quad\left|\frac{q}{p}\right|<1
$$

and

$$
\int_{0}^{\infty} f(x) \mathrm{d}_{p, q} x=(p-q) \sum_{k=-\infty}^{\infty} \frac{q^{k}}{p^{k+1}} f\left(\frac{q^{k}}{p^{k+1}}\right), \quad \text { if } \quad\left|\frac{q}{p}\right|<1
$$

provided the sums converge absolutely.

A function $f$ is said to be $(p, q)$-integrable on $[0, \infty)$, if the series

$$
\sum_{k=-\infty}^{\infty} \frac{q^{k}}{p^{k+1}} f\left(\frac{q^{k}}{p^{k+1}}\right)
$$

converges absolutely. Moreover, we assume that, $f(t)$ is a function defined on $[0, \infty)$ and satisfying $\int_{0}^{x}\left|f(t) d_{p, q} t\right|<\infty$ and also, let the partial sum of $f(t)$ be defined as

$$
\bar{s}(x)=\int_{0}^{x} f(t) \mathrm{d}_{p, q} t
$$

Next, we define the $(p, q)$ Cesàro mean of $f(x)$ as follows:

$$
t(\bar{s}(x))=\frac{1}{x} \int_{0}^{x} \bar{s}(\zeta) \mathrm{d}_{p, q} \zeta .
$$

The function $\bar{s}(x)$ is $(p, q)$-Cesàro integrable to $\ell$, if

$$
\lim _{x \rightarrow \infty} t(\bar{s}(x))=\ell
$$

Furthermore, the function $\bar{s}(x)$ is $(p, q)$-statistically Cesàro summable to $\ell$ if, for every $\epsilon>0$,

$$
\lim _{u \rightarrow \infty} \frac{1}{u} \mid\{0<x \leqq u \text { and }|t(\bar{s}(x))-\ell| \geqq \epsilon\} \mid=0 .
$$


In this case, we write

$$
\text { stat } \lim _{x \rightarrow \infty} t(\bar{s}(x))=\ell .
$$

If $\lim _{x \rightarrow \infty} \bar{s}(x)=\ell$ exists, then the relations (2.4) and (2.5) hold. However, in general, the converse is not true. To prove the oscillatory behavior and De la vallée Poussin mean based onsufficient part, we use the $(p, q)$-calculus of the above integral over $\mathbb{R}$. Such a condition is called a Tauberian condition and the resulting theorem is called a Tauberian theorem under post-quantum calculus.

For each non-negative integer $k$ we define

$$
\sigma^{(k)}(s(x))= \begin{cases}\frac{1}{x} \int_{0}^{x} \sigma^{(k-1)} s(\zeta) \mathrm{d} \zeta & (k \geq 1) \\ \int_{0}^{x} s(\zeta) \mathrm{d} \zeta & (k=0)\end{cases}
$$

An integral $\int_{0}^{\infty} f(x) \mathrm{d} x$ is said to be statistically $(C, k)$-summable to $\ell$ if and only if $\sigma^{(k)}(s(x))$ is statistically summable to $\ell$.

Remark 2.1 If $k=1$, then $(C, k)$-summabllity mean is same as the $(C, 1)$-summability mean.

Next, for $(p, q)$-calculus and for each non-negative integer $k$, we define

$$
t^{(k)}(\bar{s}(x))= \begin{cases}\frac{1}{x} \int_{0}^{x} t^{(k-1)} \bar{s}(\zeta) \mathrm{d}_{p, q} \zeta & (k \geq 1) \\ \int_{0}^{x} \bar{s}(\zeta) \mathrm{d}_{p, q} \zeta & (k=0) .\end{cases}
$$

An integral $\int_{0}^{\infty} f(x) \mathrm{d} x$ is said to be statistically $(C, k)$-summable to $\ell$ under post-quantum calculus if and only if $t^{(k)}(\bar{s}(x))$ is statistically summable to $\ell$.

Remark 2.2 If $k=1$ and $p=1$, then $(C, k)$-summabllity mean reduces $(C, 1)$-summability mean in $q$ calculus. Furthermore, if $k=1$ and $q=p=1$, then $(C, k)$-summabllity mean reduces to simply $(C, 1)$ summability mean.

Next we have, the partial sum of the function is

$$
s(x)=\int_{0}^{x} f(\zeta) \mathrm{d} \zeta
$$

and consequently,

$$
s(x)-\sigma(s(x))=v(f(x)),
$$

where

$$
v(f(x))=\frac{1}{x} \int_{0}^{x} \zeta f(\zeta) \mathrm{d} \zeta
$$

Notice that

$$
\sigma(s(x))=\frac{v(f(x))}{x} .
$$

Now, let us define for each non-negative integer $k$,

$$
v^{(k)}(f(x))= \begin{cases}\frac{1}{x} \int_{0}^{x} v^{(k-1)} \zeta f(\zeta) \mathrm{d} \zeta, & \text { for } \quad k \geq 1 \\ \int_{0}^{x} \zeta f(\zeta) \mathrm{d} \zeta, & \text { for } \quad k=0\end{cases}
$$


Here, the integral $\int_{0}^{\infty} x f(x) \mathrm{d} x$ is statistically $(C, k)$-summable to $\ell$ if and only if $v^{(k)}(f(x))$ is statistically summable to $\ell$.

Similarly, for post-quantum calculus, the partial sum of the function is

$$
\bar{s}(x)=\int_{0}^{x} f(\zeta) \mathrm{d}_{p, q} \zeta
$$

Also, we have

$$
\bar{s}(x)-t(\bar{s}(x))=(p, q) \bar{v}(f(x)),
$$

where

$$
\bar{v}(f(x))=\frac{1}{x} \int_{0}^{x} \zeta f(\zeta) \mathrm{d}_{p, q} \zeta
$$

Notice that

$$
D_{p, q}(\bar{t}(\bar{s}(x)))=\frac{\bar{v}(f(x))}{x}
$$

Now, we define for each non negative integer $k$,

$$
\bar{v}^{(k)}(f(x))= \begin{cases}\frac{1}{x} \int_{0}^{x} \bar{v}^{(k-1)} \zeta f(\zeta) \mathrm{d}_{p, q} \zeta, & \text { for } k \geq 1 \\ \int_{0}^{x} \zeta f(\zeta) \mathrm{d}_{p, q} \zeta, & \text { for } k=0\end{cases}
$$

Here, the integral $\int_{0}^{\infty} x f(x) \mathrm{d} x$ is statistically $(C, k)$-summable to $\ell$ under post-quantum calculus if and only if $\bar{v}^{(k)}(f(x))$ is summable to $\ell$.

Now recalling the De la Vallée Poussin mean (see [3]) of the integral $\int_{0}^{x} f(\zeta) \mathrm{d} \zeta$, we have

$$
\tau(s(x))=\frac{1}{(x-\lambda x)} \int_{\lambda x}^{x} s(\zeta) \mathrm{d} \zeta, \quad \lambda \in(0,1) .
$$

In the similar way, we define the De la Vallée Poussin mean for the post-quantum calculus of the integral $\int_{0}^{x} f(\zeta) \mathrm{d} \zeta$ as

$$
\tau(\bar{s}(x))=\frac{1}{(p-q) x} \int_{q x}^{p x} s(\zeta) \mathrm{d}_{p, q} \zeta, \quad(0<q<p \leq 1) .
$$

Note that, an integral $\int_{0}^{x} f(x) \mathrm{d} x$ belonging to $\mathbb{R}$ is oscillating slowly if [20]

$$
\lim _{\lambda \rightarrow 1^{-}} \limsup _{x \rightarrow \infty} \max _{\lambda x \leq \zeta \leq x}|s(\zeta)-s(x)|=0 .
$$

In the same way, under post-quantum calculus the integral $\int_{0}^{x} f(x) \mathrm{d} x$ belonging to $\mathbb{R}$ is oscillating slowly if

$$
\limsup _{x \rightarrow \infty} \max _{q x \leq \zeta \leq p x}|s(\zeta)-s(x)|=0 .
$$




\section{Main results}

Theorem 3.1 If $\bar{s}(x)$ is $(p, q)$-statistically $(C, 1)$-summable to $\ell$ and $\bar{s}(x)$ is $(p, q)$-oscillating slowly, then $\bar{s}(x) \rightarrow \ell$, as $x \rightarrow \infty$.

The proof of this theorem requires the following lemmas

Lemma 3.2 The sequence of partial sum $\bar{s}(x)$ of an integrable function $f(x)$ over $\mathbb{R}$ is $(p, q)$-oscillating slowly if and only if $\bar{v}(f(x))$ is bounded and oscillating slowly.

Proof Let $\bar{s}(x)$ be $(p, q)$-oscillating slowly. Initially, let us show that $\bar{v}(f(x))=O(1)$ as $x \rightarrow \infty$.

We have

$$
\int_{0}^{x} w f(w) \mathrm{d}_{p, q} w=\sum_{i=0}^{\infty} \int_{x / 2^{i+1}}^{x / 2^{i}} w f(w) \mathrm{d}_{p, q} w .
$$

Now it follows from Eq. (2.7) that

$$
\begin{aligned}
\int_{\alpha}^{\beta} w f(w) \mathrm{d}_{p, q} w= & \int_{\alpha}^{\beta} w \bar{s}(w) \mathrm{d}_{p, q} w \\
= & {\left[w(\bar{s}(w))_{\alpha}^{\beta}-\int_{\alpha}^{\beta} \bar{s}(w) \mathrm{d}_{p, q} w\right] } \\
= & -\int_{\alpha}^{\beta} \bar{s}(w) \mathrm{d}_{p, q} w+\beta(\bar{s}(\beta))-\alpha(\bar{s}(\alpha))-\alpha(\bar{s}(\beta))+\alpha(\bar{s}(\beta)) \\
& -\int_{\alpha}^{\beta} \bar{s}(w) \mathrm{d}_{p, q} w+(\beta-\alpha) \bar{s}(\beta)+\alpha(\bar{s}(\beta)-\bar{s}(\alpha)) \\
= & -\int_{\alpha}^{\beta}(\bar{s}(w)-\bar{s}(\beta)) \mathrm{d}_{p, q} w+\alpha(\bar{s}(\beta)-\bar{s}(\alpha)) \\
= & (\beta-\alpha) \max _{q \alpha \leq x \leq p \beta}|\bar{s}(x)-\bar{s}(\beta)|+\alpha|\bar{s}(\beta)-\bar{s}(x)|
\end{aligned}
$$

If we choose $\beta=x / 2^{i}$ and $\beta / \alpha \leq 2$, we obtain

$$
\int_{0}^{x} w f(w) \mathrm{d}_{p, q} w \leq A \sum_{i=0}^{\infty} \frac{x}{2^{i}}=O(x), \quad \text { as } \quad x \rightarrow \infty .
$$

Now we have to show that $t(\bar{s}(x))$ is oscillating slowly. Since

$$
D_{p, q}(\bar{t}(\bar{s}(x)))=\frac{\bar{v}(f(x))}{x},
$$

we get

$$
\begin{aligned}
|t(\bar{s}(\zeta))-t(\bar{s}(x))| & =\left|\int_{x}^{\zeta} \bar{t}(\bar{s}(w)) \mathrm{d}_{p, q} w\right| \\
& =\left|\int_{x}^{\zeta} f(w) \mathrm{d}_{p, q} w\right| \\
& \leq C \int_{x}^{\zeta} \frac{\mathrm{d}_{p, q} w}{w} \\
& =C \log (\zeta / x), \text { for any } q x \leq \zeta \leq p x
\end{aligned}
$$

Clearly, we have

$$
\max _{q x \leq \zeta \leq p x}|t(\bar{s}(\zeta))-t(\bar{s}(x))| \leq C
$$


and

$$
\lim \sup _{x \rightarrow \infty} \max _{q x \leq \zeta \leq p x} \mid t(\bar{s}(\zeta)-t(\bar{s}(x)) \mid=0 .
$$

That implies $\bar{v}(f(x))$ is oscillating slowly by Kronecker identity.

To prove the converse part, suppose that $\bar{v}(f(x))$ is bounded and oscillating slowly. The boundedness of $\bar{v}(f(x))$ implies that $t(\bar{s}(x))$ is oscillating slowly. Since $\bar{v}(f(x))$ is oscillating slowly, so $\bar{s}(x)$ is oscillating slowly by Kronecker identity. This establishes Lemma 3.2.

Lemma 3.3 For $0<q<p \leqq 1$,

$$
\bar{s}(x)-t(\bar{s}(x))=\frac{q}{(p-q)}(t(\bar{s}(x))-\bar{s}(x))-\frac{q}{(p-q) x} \int_{q x}^{p x}(\bar{s}(x)-\bar{s}(\zeta)) \mathrm{d}_{p, q} \zeta .
$$

Proof We have by De la Vallée Poussin mean of $\bar{s}(x)$,

$$
\begin{aligned}
\tau(\bar{s}(x)) & =\frac{q}{(p-q) x} \int_{q x}^{p x} \bar{s}(\zeta) \mathrm{d}_{p, q} \zeta \\
& =\frac{q}{x(p-q)}\left(\int_{0}^{q x} \bar{s}(\zeta) \mathrm{d} \zeta-\int_{0}^{p x} \bar{s}(\zeta) \mathrm{d} \zeta\right) .
\end{aligned}
$$

Again, since

$$
t(\bar{s}(x))=\frac{1}{x} \int_{0}^{q x} \bar{s}(\zeta) \mathrm{d}_{p, q} \zeta
$$

and

$$
t(\bar{s}(x))=\frac{1}{x} \int_{0}^{p x} \bar{s}(\zeta) \mathrm{d}_{p, q} \zeta
$$

we have

$$
\begin{aligned}
\tau(\bar{s}(x)) & =\frac{q^{2}}{(p-q)^{2}} t(\bar{s}(x))-\frac{1}{(p-q)^{2}} t(\bar{s}(x)) \\
& =\left(1+\frac{1}{(p-q)}\right)^{2} t(\bar{s}(x))-\frac{1}{(p-q)^{2}} t(\bar{s}(x)) .
\end{aligned}
$$

Now

$$
\begin{aligned}
\tau(s(x))-t(\bar{s}(x))= & \frac{1}{(p-q)^{2}} t(\bar{s}(x))+\frac{2}{(p-q)} t(\bar{s}(x)) \\
& -\frac{1}{(p-q)^{2}} \sigma(s(x)) .
\end{aligned}
$$

Subtracting $t(\bar{s}(x))$ from the identity, also

$$
\bar{s}(x)=\tau(\bar{s}(x))-\frac{1}{(p-q) x} \int_{q x}^{p x}(\bar{s}(\zeta)-\bar{s}(x)) \mathrm{d}_{p, q} \zeta,
$$

we have

$$
\begin{aligned}
\bar{s}(x)-t(\bar{s}(x))= & \tau(\bar{s}(x)-t(\bar{s}(x)) \\
& -\frac{q}{(p-q) x} \int_{q x}^{p x}(\bar{s}(\zeta)-\bar{s}(x)) \mathrm{d}_{p, q} \zeta .
\end{aligned}
$$

From Eqs. (3.2) and (3.3), we get

$$
\begin{aligned}
\bar{s}(x)-t(\bar{s}(x))= & \frac{1}{(p-q)^{2}}(t(\bar{s}(x))-\sigma(\bar{s}(x))) \\
& +\frac{2}{(p-q)} t(\bar{s}(x))-\frac{q}{(p-q) x} \int_{q x}^{p x}(\bar{s}(\zeta)-\bar{s}(x)) \mathrm{d}_{p, q} \zeta .
\end{aligned}
$$

This establish Lemma 2. 


\section{Proof of Theorem 3.1.}

Proof Let $\bar{s}(x)$ be statistically $(C, 1,1)$-summable to $\ell$, this implies $t(\bar{s}(x))$ is $(C, 1)$-summable to $\ell$. Now from Eq. (2.7), we have $\bar{v}(f(x))$ is statistically $(C, 1)$-summable to zero. Thus, by Lemma 3.2, $\bar{v}(f(x))$ is oscillating slowly. Furthermore, by Lemma 3.3, we get

$$
\begin{aligned}
\bar{v}(f(x))-t(\bar{v}(f(x)))= & \frac{q}{(p-q)^{2}}((t(\bar{v}(f(x))))-t(\bar{v}(f(x)))) \\
& +\frac{2}{(p-q)} t(\bar{v}(f(x)))-\frac{q}{(p-q) x} \\
& \cdot \int_{q x}^{p x}(\bar{v}(f(\zeta))-\bar{v}(f(x))) \mathrm{d}_{p, q} \zeta .
\end{aligned}
$$

Next, by (3.4)

$$
\begin{aligned}
|\bar{v}(f(x))-t(\bar{v}(f(x)))| \leq & \frac{1}{(p-q)^{2}}|t(\bar{v}(f(x)))-t(\bar{v}(f(x)))| \\
& +\frac{2}{(p-q)}|t(\bar{v}(f(x)))| \\
& +\max _{q x \leq \zeta \leq p x}|\bar{v}(f(\zeta))-\bar{v}(f(x))| .
\end{aligned}
$$

Now taking lim sup on both sides of Eq. (3.5) as $x \rightarrow \infty$, we obtain

$$
\begin{aligned}
\lim \sup _{x \rightarrow \infty} \mid \bar{v}(f(x))- & t(\bar{v}(f(x))) \mid \\
\leq & \lim \sup _{x \rightarrow \infty} \frac{1}{(p-q)^{2}}|t(\bar{v}(f(x)))-t(\bar{v}(f(x)))| \\
& +\lim \sup _{x \rightarrow \infty} \frac{2}{(p-q)}|t(\bar{v}(f(x)))| \\
& +\lim _{x \rightarrow \infty} \sup _{x \rightarrow \zeta \leq p x}|\bar{v}(f(\zeta))-\bar{v}(f(x))| .
\end{aligned}
$$

Furthermore, as $t(\bar{v}(f(x)))$ converges, the first and second terms in the right-hand side of Eq. (3.6) must vanish.

This implies

$$
\lim \sup _{x \rightarrow \infty}|\bar{v}(f(x))-t(\bar{v}(f(x)))| \leq \lim \sup _{x \rightarrow \infty} \max _{q \leq \zeta \leq p x}|\bar{v}(f(\zeta))-\bar{v}(f(x))| .
$$

As $0<q<p \leq 1$ in (3.7), so we get

$$
\lim \sup _{x \rightarrow \infty}|\bar{v}(f(x))-t(\bar{v}(f(x)))| \leq 0 .
$$

It implies that, $\bar{v}(f(x))=o(1)$ as $x \rightarrow \infty$. Since $\bar{s}(x)$ is statistically summable to $\ell$ by Cesàro mean and $\bar{v}(f(x))=o(1)$ as $x \rightarrow \infty$, so $\lim _{x \rightarrow \infty} \bar{s}(x)=\ell$.

Theorem 3.4 Let $p_{n}=q_{n}=1$, if $s(x)$ is statistically $(C, 1)$-summable to $\ell$ and $s(x)$ is oscillating slowly, then $s(x) \rightarrow \ell$, as $x \rightarrow \infty$.

Proof Let $s(x)$ is statistically $(C, 1)$-summable to $\ell$, this implies $\sigma(s(x))$ is $(C, 1)$-summable to $\ell$. Now from Eq. (2.6), we have $v(f(x))$ is statistically $(C, 1)$-summable to zero. Thus, by Lemma 3.2, $v(f(x))$ is oscillating slowly. Again by Lemma 3.3, we get 


$$
\begin{aligned}
v(f(x))-\sigma(v(f(\lambda x)))= & \frac{1}{(\lambda-1)^{2}}((\sigma(v(f(\lambda x))))-\sigma(v(f(x)))) \\
& +\frac{2}{(\lambda-1)} \sigma(v(f(\lambda x)))-\frac{1}{(\lambda x-x)} \\
& \cdot \int_{x}^{\lambda x}(v(f(\zeta))-v(f(x))) \mathrm{d} \zeta .
\end{aligned}
$$

Next, by (3.8)

$$
\begin{aligned}
|v(f(x))-\sigma(v(f(x)))| \leq & \frac{1}{(\lambda-1)^{2}}|\sigma(v(f(\lambda x)))-\sigma(v(f(x)))| \\
& +\frac{2}{(\lambda-1)}|\sigma(v(f(\lambda x)))|+\max _{x \leq \zeta \leq \lambda x}|v(f(\zeta))-v(f(x))| .
\end{aligned}
$$

Now taking lim sup on both sides of Eq. (3.9) as $x \rightarrow \infty$, we obtain

$$
\begin{aligned}
\lim \sup _{x \rightarrow \infty} \mid v(f(x))- & \sigma(v(f(x))) \mid \\
\leq & \lim \sup _{x \rightarrow \infty} \frac{1}{(\lambda-1)^{2}}|\sigma(v(f(\lambda x)))-\sigma(v(f(x)))| \\
& +\lim \sup _{x \rightarrow \infty} \frac{2}{(\lambda-1)}|\sigma(v(f(\lambda x)))| \\
& +\lim \sup _{x \rightarrow \infty} \max _{x \leq \zeta \leq \lambda x}|v(f(\zeta))-v(f(x))| .
\end{aligned}
$$

Furthermore, as $\sigma(v(f(\lambda x)))$ converges, the first and second terms in the right-hand side of Eq. (3.10) must vanish.

This implies

$$
\begin{aligned}
& \lim \sup _{x \rightarrow \infty}|v(f(x))-\sigma(v(f(x)))| \\
& \leq \lim \sup _{x \rightarrow \infty} \max _{x \leq \zeta \leq \lambda x}|v(f(\zeta))-v(f(x))| .
\end{aligned}
$$

As $\lambda \rightarrow 1^{+}$in (3.11), so we get

$$
\lim \sup _{x, y \rightarrow \infty}|v(f(x))-\sigma(v(f(x)))| \leq 0 .
$$

It implies that $v(f(x))=o(1)$ as $x \rightarrow \infty$. Since $s(x)$ is statistically summable to $\ell$ by Cesàro mean and $v(f(x))=o(1)$ as $x \rightarrow \infty$, so $\lim _{x \rightarrow \infty} s(x)=\ell$.

Corollary 3.5 If $\bar{s}(x)$ is statistically $(C, k)$-summable to $\ell$ and $\bar{s}(x)$ is $(p, q)$-oscillating slowly, then $\bar{s}(x) \rightarrow \ell$ as $x \rightarrow \infty$.

Proof By Lemma 3.2, $\bar{s}(x)$ is oscillating slowly and also $t^{(k)}(\bar{s}(x))$ is oscillating slowly. Furthermore, from Theorem $3.1, \bar{s}(x)$ being statistically $(C, k)$-summable to $\ell$, so

$$
\text { stat } \lim _{x \rightarrow \infty} t^{(k)}(\bar{s}(x))=\ell .
$$

Next, from the definition,

$$
t^{(k)}(\bar{s}(x))=t^{(1)}(\bar{s}(x))\left(t^{(k-1)}(\bar{s}(x))\right) .
$$

Clearly, Eqs. (3.12) and (3.13) implies that $\bar{s}(x)$ is statistically $(C, k-1)$-summable to $\ell$. Again by Lemma 3.2, $t^{(k-1)}(\bar{s}(x))$ is also oscillating slowly.

Thus, Theorem 3.1 implies

$$
\lim _{x \rightarrow \infty} t^{(k-1)}(\bar{s}(x))=\ell .
$$

Continuing in this way, we get $\lim _{x \rightarrow \infty}(\bar{s}(x))=\ell$. 
Theorem 3.6 If $\bar{s}(x)$ is $(p, q)$-statistically $(C, 1)$-summable to $\ell$ and $\bar{v}(f(x))$ is $(p, q)$-oscillating slowly, then $\bar{s}(x) \rightarrow \ell(x \rightarrow \infty)$.

Proof The proof of this theorem is similar to the proof of Theorem 3.1.

Theorem 3.7 If $s(x)$ is statistically $(C, 1)$-summable to $\ell$ and $v(f(x))$ is oscillating slowly, then $s(x) \rightarrow \ell$ as $x \rightarrow \infty$.

Proof The proof of this theorem is similar to the proof of Theorem 3.4.

Corollary 3.8 If $\bar{s}(x)$ is statistically $(C, k)$-summable to $\ell$ and $\bar{v}(f(x))$ is oscillating slowly, then $\bar{s}(x) \rightarrow \ell$ as $x \rightarrow \infty$.

Proof As $\bar{v}(f(x))$ is oscillating slowly, setting $\bar{v}(f(x))$ in place of $\bar{s}(x) ; t^{(k)}(\bar{v}(f(x)))$ is oscillating slowly by Lemma 3.2. Again as $\bar{v}(f(x))$ is statistically $(C, k)$ summable to $\ell$, so Theorem 3.6 implies

$$
\text { stat } \lim _{x \rightarrow \infty} t^{(k)}(\bar{v}(f(x)))=\ell
$$

Next by definition,

$$
\lim _{x \rightarrow \infty} t^{(k)}(\bar{v}(f(x)))=t^{(1)}(\bar{v}(f(x))) t^{(k-1)}(\bar{v}(f(x))) .
$$

From (3.14) and (3.15), it is clear that $\bar{v}(f(x))$ is statistically $(C, k-1)$-summable to $\ell$. Moreover, by Lemma $3.2, \sigma^{k-1}(\bar{v}(f(x)))$ being oscillating slowly, by Theorem -3.6, we have $\lim _{x \rightarrow \infty} t^{k-1}(\bar{v}(f(x)))=\ell$. Continuing in this way, we obtain $\lim _{x \rightarrow \infty} \bar{v}(f(x))=\ell$.

\section{Conclusion}

Tauberian theorems for single sequences as well as for functions of single variable have achieved a high degree of development; however, it is still in its infancy for double sequences and functions of two or more variables. The result established here that the statistical versions of Tauberian theorems for a real-valued continuous function of one variable under the post-quantum calculus (or $(p, q)$-calculus) of integrals via statistical Cesàro summability mean generalizes some earlier existing Tauberian theorems for the function of a single variable in quantum calculus (or $(q)$-calculus) via classical Cesàro summability mean. Further, it will be encouraging if one can extend the result for functions of two or more variables using the post-quantum calculus (or $(p, q)$ calculus) of integrals via the statistical Cesàro mean and so also for other different statistical versions of summability means.

Open Access This article is licensed under a Creative Commons Attribution 4.0 International License, which permits use, sharing, adaptation, distribution and reproduction in any medium or format, as long as you give appropriate credit to the original author(s) and the source, provide a link to the Creative Commons licence, and indicate if changes were made. The images or other third party material in this article are included in the article's Creative Commons licence, unless indicated otherwise in a credit line to the material. If material is not included in the article's Creative Commons licence and your intended use is not permitted by statutory regulation or exceeds the permitted use, you will need to obtain permission directly from the copyright holder. To view a copy of this licence, visit http://creativecommons.org/licenses/by/4.0/.

\section{References}

1. Çanak, İ.; Totur, Ü.: A Tauberian theorem for Cesàro summability of integrals. Appl. Math. Lett. 24, 391-395 (2011)

2. Çanak, İ.; Totur, Ü.: Some Tauberian conditions for Cesàro summability method. Math. Slovaca 62, 271-280 (2012)

3. Çanak, İ.; Totur, Ü.; Sezer, S.A.: Cesàro integrability and Tauberian theorems in quantum calculus. An. Ştiint. Univ. Al. I. Cuza Iaşi. Mat. (N.S.) LXIV, 9-19 (2018)

4. Das, A.A.; Jena, B.B.; Paikray, S.K.; Jati, R.K.: Statistical deferred weighted summability and associated Korovokin-type approximation theorem. Nonlinear Sci. Lett. A 9, 238-245 (2018)

5. Fast, H.: Sur la convergence statistique. Colloq. Math. 2, 241-244 (1951)

6. Hardy, G.H.: Divergent Series. Clarendon Press, Oxford (1949)

7. Hardy, G.H.; Littlewood, J.E.: Tauberian theorems concerning power series and Dirichlet's series whose coeffcients are positive. Proc. Lond. Math. Soc. 13, 174-191 (1914) 
8. Jena, B.B.; Paikray, S.K.: Product of statistical probability convergence and its applications to Korovkin-type theorem. Miskolc Math. Notes 20, 969-984 (2019)

9. Jena, B.B.; Paikray, S.K.; Misra, U.K.: A Proof of Tauberian theorem for Cesàro summability method. Asian J. Math. Comput. Res 8, 272-276 (2016)

10. Jena, B.B.; Paikray, S.K.; Misra, U.K.: A Tauberian theorem for double Cesàro summability method. Int. J. Math. Math. Sci. 2016, 1-4 (2016). Article ID 2431010

11. Jena, B.B.; Paikray, S.K.; Misra, U.K.: Inclusion theorems on general convergence and statistical convergence of $(L, 1,1)$ summability using generalized Tauberian conditions. Tamsui Oxf. J. Inf. Math. Sci. 31, 101-115 (2017)

12. Jena, B.B.; Paikray, S.K.; Misra, U.K.: Statistical deferred Cesàro summability and its applications to approximation theorems. Filomat 32, 2307-2319 (2018)

13. Jena, B.B.; Paikray, S.K.; Misra, U.K.: Approximation of periodic functions via statistical $B$-summability and its applications to approximation theorems. Indian J. Industr. Appl. Math. 10, 71-86 (2019)

14. Jena, B.B.; Paikray, S.K.; Parida, P.; Dutta, H.: Results on Tauberian theorem for Cesàro summable double sequences of fuzzy numbers. Kragujevac J. Math. 44, 495-508 (2020)

15. Jena, B.B.; Paikray, S.K.; Mohiuddine, S.A.; Mishra, V.N.: Relatively equi-statistical convergence via deferred Nörlund mean based on difference operator of fractional-order and related approximation theorems. AIMS Math. 5, 650-672 (2020)

16. Knopp, K.: Limitierungs-Umkehrsätze für Doppelfolgen. Math. Z 45, 573-589 (1939)

17. Landau, E.: Über die Bedeutung einiger neuerer Grenzwertsätze der Herren Hardy and Axer. Prace Mat. Fiz. 21, 97-177 (1910)

18. Landau, E.: Über einen Satz des Herrn Littlewood. Palermo Rend. 35, 265-276 (1913)

19. Móricz, F.: Tauberian theorems for Cesàro summable double sequences. Studia Math. 110, 83-96 (1994)

20. Móricz, F.: Tauberian theorem for Cesàro summable double integrals over $R_{+}^{2}$. Stud. Math. 138, 41-52 (2002)

21. Parida, P.; Paikray, S.K.; Dutta, H.; Jena, B.B.; Dash, M.: Tauberian theorems for Cesàro summability of $n$-th sequences. Filomat 31, 3993-4004 (2018)

22. Parida, P.; Paikray, S.K.; Dash, M.; Misra, U.K.: Degree of approximation by product $\left(\bar{N}, p_{n}, q_{n}\right)(E, q)$ summability of Fourier series of a signal belonging to Lip $(\alpha, r)$-class. TWMS J. App. Eng. Math. 9, 901-908 (2019)

23. Pradhan, T.; Paikray, S.K.; Jena, B.B.; Dutta, H.: Statistical deferred weighted $\mathcal{B}$-summability and its applications to associated approximation theorems. J. Inequal. Appl. 2018, 1-21 (2018). Article Id: 65

24. Pradhan, T.; Jena, B.B.; Paikray, S.K.; Dutta, H.; Misra, U.K.: On approximation of the rate of convergence of Fourier series in the generalized Hölder metric by Deferred Nörlund mean. Afr. Mat. 30, 1119-1131 (2019)

25. Schmidt, R.: Über divergente Folgen und lineare Mittelbildungen. Math. Z. 22, 89-152 (1925)

26. Srivastava, H.M.; Jena, B.B.; Paikray, S.K.: A certain class of statistical probability convergence and its applications to approximation theorems, Appl. Anal. Discrete Math. (in press) (2020)

27. Srivastava, H.M.; Jena, B.B.; Paikray, S.K.; Misra, U.K.: A certain class of weighted statistical convergence and associated Korovkin type approximation theorems for trigonometric functions. Math. Methods Appl. Sci. 41, 671-683 (2018)

28. Srivastava, H.M.; Jena, B.B.; Paikray, S.K.; Misra, U.K.: Generalized equi-statistical convergence of the deferred Nörlund summability and its applications to associated approximation theorems. Rev. R. Acad. Cienc. Exactas Fís. Nat. Ser. A Mat. (RACSAM) 112, 1487-1501 (2018)

29. Srivastava, H.M.; Jena, B.B.; Paikray, S.K.; Misra, U.K.: Deferred weighted $\mathcal{A}$-statistical convergence based upon the $(p, q)$-Lagrange polynomials and its applications to approximation theorems. J. Appl. Anal. 24, 1-16 (2018)

30. Srivastava, H.M.; Jena, B.B.; Paikray, S.K.; Misra, U.K.: Statistically and relatively modular deferred-weighted summability and Korovkin-type approximation theorems. Symmetry 11, 1-20 (2019). Article Id:448

31. Srivastava, H.M.; Jena, B.B.; Paikray, S.K.: Deferred Cesàro statistical probability convergence and its applications to approximation theorems. J. Nonlinear Convex Anal. 20, 1777-1792 (2019)

32. Steinhaus, H.: Sur la convergence ordinaire et la convergence asymptotique. Colloq. Math. 2, $73-74$ (1951)

33. Tauber, A.: Ein satz der Theorie der unendlichen Reihen. Monatsh. Math. 8, 273-277 (1897)

34. Totur, Ü.: Classical Tauberian theorems for $(C, 1,1)$-summability method, An. Stiinu. Univ. Al. I. Cuza Iasi. Mat. (2014). https://doi.org/10.2478/aicu-2014-0010

Publisher's Note Springer Nature remains neutral with regard to jurisdictional claims in published maps and institutional affiliations. 